\title{
Development and Validation of a Nomogram for Predicting Recurrence in Patients with AF Who Underwent Ablation within
}

\section{one year}

\author{
Yun Cheng$^{1}$ and Xue Feng ${ }^{1}$ \\ ${ }^{1}$ First Affiliated Hospital of Soochow University
}

June 15, 2020

\begin{abstract}
Background:With the improvement of radiofrequency catheter ablation technology, an increasing number of patients with atrial fibrillation $(\mathrm{AF})$ choose it as the treatment option. However, the individual recurrence rate after ablation is difficult to accurately predict. Objective:This study aimed to develop and validate an effective prognostic nomogram for predicting long-term recurrence of patients with AF who underwent ablation. Methods: We conducted a retrospective single-center cohort study of 493 patients with AF from five wards in The First Affiliated Hospital of Soochow University from January 1, 2015 to December 31, 2019. Three quarters of patients $(n=371)$ were randomly assigned to the training cohort, and the rest $(\mathrm{n}=122)$ were assigned to the validation cohort. Univariate and multivariate cox regression analysis was performed using $\mathrm{R}$ software version 3.6.2 to prognostic variables for recurrence and develop a nomogram. The C index, ROC, calibration curve, Greenwood-D'Agostino-Nam, and DCA were used for verification in the modeling cohort and the verification cohort respectively for validation. Results: Multivariate cox regression analysis shown that 6 independent predictors were identified: age, female, AF duration, AF type, coronary artery disease, left atrial diameter. And these predictors were entered into the nomogram, which shown favorable discrimination and calibration both in the training cohort and validation cohort. Conclusion: The proposed nomogram can accurately predict recurrence of patients after AF ablation. Compared to the CHA2DS2-VASc score, clinicians can promote individual-oriented therapy and disease management by using this tool.
\end{abstract}

\section{Hosted file}

Development and Validation of a Nomogram for Predicting Recurrence in Patients with AF Who Underwent Ab available at https://authorea.com/users/333785/articles/459846-development-and-validationof-a-nomogram-for-predicting-recurrence-in-patients-with-af-who-underwent-ablationwithin-one-year 